\title{
Impact assessment of effectiveness of thiourea and THAM for corrosion process on carbon steel in calcium chloride environment
}

\author{
Szymon Malinowski, Justyna Jaroszyńska-Wolińska \\ Faculty of Civil Engineering \& Architecture, Lublin University of Technology, \\ e-mail: s.malinowski@pollub.pl,
}

\begin{abstract}
The main objective of this paper is to present a comparison of the corrosion inhibiting effect of thiourea and THAM (tri(hydroxymethyl)aminomethane) on carbon steel samples immersed in calcium chloride solutions. The testing has shown that to inhibit the corrosive action of the calcium chloride solution, the thiourea solution can be successfully replaced by the THAM solution. The study was performed with the use of st $3 \mathrm{~s}$, structural and reinforcing steel. In the case of st3s steel samples, the application of the $0.01 \%$ THAM solution causes a decrease of its weight loss by about $60 \%$. The application of a 10-times stronger THAM solution causes an increase of the corrosion inhibition on the tested samples by about $20 \%$. A combined protective action of the thiourea and THAM solutions brings about an increase of the corrosion process inhibition by about $70 \%$ both for the st $3 \mathrm{~s}$ and the structural samples. The $0.01 \%$ and $0.1 \%$ THAM solutions applied to inhibit the corrosion process on structural samples cause an increase of the corrosion protection by about $50 \%$ and $15 \%$ respectively.
\end{abstract}

Keywords: corrosion inhibitor, thiourea, THAM, carbon steel, anticorrosive properties.

\section{Introduction}

Corrosion problems are essential for numerous branches of the industry. Therefore, considerable attention is presently paid to investigations into new non-toxic and ecofriendly corrosion inhibitors. The main objective of such investigations is to minimize or inhibit the corrosion processes [1].

Corrosion processes result from the effect of the redox (reduction-oxidation) reaction on metal surfaces. It can be analyzed into two main half electrochemical reactions. An oxidation process occurs on the anode and is related to the metal dissolution and its losing positive ions to the electrolyte. Free electrons that form in those processes are absorbed in the reduction process of hydrogen ions that takes place on the cathode [2]. Corrosion processes make one of the most severe problems of many industries. This is particularly conspicuous in the case of the oil and gas industry, where the corrosion on surfaces of the equipment and processing facilities causes leaks in tanks, casings or pipelines. In the petroleum industry, the corrosion problem the most severely shows in the case of pipelines [3]. It results from the application acid solutions to e.g. chemical processing, petroleum production or acid cleaning [4]. Hydrochloric acid $(\mathrm{HCl})$ is one of the acid solutions that are most widely used for those purposes, which exposes steel and ferrous alloy materials of the equipment to the corrosion processes. The most effective method for protecting the equipment is to use corrosion inhibitors [5], especially in closed loop systems [6]. The inhibitors are substances, whose very small amounts added to the corrosive solution can 
decrease the rate of the destructive reaction that occurs on the metal surface in the presence of the corrosive agent [7]. Considering the protective mechanism, corrosion inhibitors can be classified into anodic, cathodic and mixed ones. Anodic inhibitors reduce the corrosion rate by inhibiting the dissolution of steel. Cathodic corrosion inhibitors reduce the corrosion process by decreasing the corrosion potential, while mixed corrosion inhibitors act both on the anodic and cathodic corrosion reactions. Among inorganic compounds, the group of anodic corrosion inhibitors includes e.g. calcium nitrate, sodium nitrate, sodium benzoate, while in the group of cathodic corrosion inhibitors there are phosphates, silicate and polyphosphates [8]

Corrosion inhibitors play very important role in protecting various kinds of steel. For that purpose both organic and inorganic compounds can be applied. The structure of organic corrosion inhibitors includes either heteroatoms such as phosphorus, nitrogen, sulfur or oxygen, $\pi$ bonds [11], and also aromatic rings [6]. Many organic compounds that include amines, amides, nitrogen-containing heterocycles, nitriles, imminium salts, triazoles, pyridine and its derivates, quanoline derivates, thiourea derivates, thiosemicarbazide, thiocyanates can be used as corrosion inhibitors. The metal dissolution process (the anodic reaction) and hydrogen evaluation (the cathodic reaction) can be retarded by compounds containing nitrogen and acetylenic alcohol by forming a film on the metal surface [3]. When organic compounds are used as corrosion inhibitors, the below given four types of adsorption can occur:

a. Electrostatic attraction between the charged molecules and the charged metal

b. Interaction of uncharged electron pairs in the molecule with the metal

c. Interaction of $\pi$ electrons with the metal

d. Combination of (a) and (c) [7].

Organic corrosion inhibitors get adsorbed on the protected metal surface and this way a thin film of inhibitor molecules forms. This film acts as a barrier and separates the metal surface from the corrosive medium. Adsorption of corrosion inhibitor molecules depends on some factors like the metal characteristic and its surface charge, the adsorption method, chemical structure of the corrosion inhibitor and the corrosion solution type [9].

One of many groups of corrosion inhibitors are surfactants. They are surface active agents that can improve dispersibility of corrosion inhibitors in acid solutions, while wetting the surface of the metallic material [3]. Both ionic and nonionic surfactants are successfully used for the corrosion protection of metallic surfaces. Nonionic surfactants have no charge groups in their polar heads and are known for their numerous applications including the corrosion inhibition. Aside with many valuable properties, their chief advantage is the low cost of their of production [1]. Presently, some inhibitors are commercially available and among them: nonylphenyl ethoxylate [3], sodium monofluorophosphate [9].

Plant extracts make a very interesting group of corrosion inhibitors. They are low-cost and eco-friendly inhibitors that can be obtained by a simple extraction or a biodegradable process. Among such inhibitors there are plant extracts of Nypa fruticans, Menthe pulegium, Phylanthus amarus, Occium virdis, Murraya koenigii and Ananas comosus. The corrosion inhibiting action of those plants extracts results from the presence of complex organic species such as tannins, alkaloids, carbohydrates, aminoacids or proteins [10].

Another popular group of corrosion inhibitors are polymers such as polyamine derivatives, polyvinylamide derivates, polyaspartates and other polyaminoacids, polyanilines, polycarboxylates/polycarboxylic acids, phosphate esters of polymeric corrosion inhibitors, polysulfides and polysaccharides. They form a thin layer of polymer on the metal surface and prevent the formation of corrosion cells [9] 
Recent years have brought a growing popularity of antibiotics used as corrosion inhibitors. All kinds of them can be used for that purpose and among them: $\beta$-lactams, quinolones, tetracyclines, macrolides, lincosamides, sulphonoamides, aminoglycosides and aminophenols [11].

As thiourea is a very toxic compound, it seems necessary to look for some new corrosion inhibitors that would be less noxious for humans. Another objective is continuous enhancement of the corrosion inhibition process, which includes the search for compounds with suitable functional groups in their structure. Therefore, the experimental part of the presented research includes testing of anticorrosive properties of THAM with an amine group with free pair of electrons in its structure and the thiourea solution.

\section{Materials and methods}

Samples of st3s steel, structural steel and reinforcing steel have been used for the testing. Their percentage composition is presented in Table 1. Each of them includes carbon, manganese, phosphorus and sulfur. The st $3 \mathrm{~s}$ steel and the reinforcing steel do not include any addition of silica and nitrogen, respectively.

Table 1. Percentage composition of the tested steel samples

\begin{tabular}{llllllll}
\hline Sample & Steel grade & $\mathrm{C}, \%$ & $\mathrm{Mn}, \%$ & $\mathrm{Si}, \%$ & $\mathrm{P}, \%$ & $\mathrm{~S}, \%$ & $\mathrm{~N}, \%$ \\
\hline st3s & $\mathrm{S} 235 \mathrm{JRG} 2$ & 0.20 & 1.40 & - & 0.045 & 0.045 & 0.09 \\
reinforcing & 18G2-b & $0.15-0.22$ & $1.0-1.5$ & $0.20-0.55$ & 0.050 & 0.050 & - \\
structural & $\mathrm{S} 235 \mathrm{jRh}$ & 0.13 & 0.34 & 0.01 & 0.012 & 0.024 & 0.004 \\
\hline
\end{tabular}

The corrosion process has been realized in the $5 \%$ solution of calcium chloride in the presence of the $0.001 \%$ thiourea solution, the $0.1 \%$ and $0.01 \%$ THAM solutions, a mixture of the $0.01 \%$ THAM solution and the $0.1 \%$ phosphorous acid solution as well as a mixture of the $0.001 \%$ thiourea solution and the $0.01 \%$ THAM solution used as the corrosion inhibitors. Quantities of the added inhibitor solutions are given in Table 2. According to the literature data [3], an addition of the phosphorous acid solution in combined action with the THAM solution should enhance the corrosion resistance of the tested materials.

Table 2. Quantatitive summary of solutions added for testing the corrosion inhibitors

\begin{tabular}{lllllll}
\hline & $1\left[\mathrm{~cm}^{3}\right]$ & $2\left[\mathrm{~cm}^{3}\right]$ & $3\left[\mathrm{~cm}^{3}\right]$ & $4\left[\mathrm{~cm}^{3}\right]$ & $5\left[\mathrm{~cm}^{3}\right]$ & $6\left[\mathrm{~cm}^{3}\right]$ \\
\hline $0.1 \%$ thiourea solution & 0 & 7 & 0 & 0 & 0 & 7 \\
$0.1 \%$ THAM solution & 0 & 0 & 7 & 70 & 7 & 7 \\
$1 \%$ phosphorous solution & 0 & 0 & 0 & 0 & 7 & 0 \\
\hline
\end{tabular}

In order to obtain reliable results, two measuring methods - the gravimetric method and the spectrophotometric one - have been independently applied in the testing process. The gravimetric method has been realized in two distinct steps. In the first one, the steel samples have been prepared for the exposure to aggressive corrosive conditions. The steel surface has been cleaned with the use of sandpaper, and in the case of structural steel, the zinc coat has been removed. Prior to putting the samples into the corrosive solution, all of them have been skimmed, dried and weighted. The other testing step has been directly related to the preparation of the corrosive solution by dissolving of adequate salts and adding adequate amounts of the corrosion inhibitor solution. Next, the steel samples have been placed in this solution for 24 hours. After the lapse of those 24 hours, the tested steel 
samples have been washed with distilled water, thoroughly dried and reweighted. On the basis of the obtained mass difference, the corrosion inhibiting efficiency has been calculated using the below formula:

$$
E=\left(1-\Delta m_{\text {inh }} / \Delta m_{0}\right) * 100 \%
$$

where: $E$ - efficiency of the corrosion inhibition process [\%], $\Delta m_{i n h}-$ weight loss in the presence of a corrosion inhibitor $[\mathrm{g}], \Delta m_{0}$ - weight loss with no corrosion inhibitor applied [g].

The spectrophotometric method has been applied because of its high sensitivity and high accuracy of the ferric ion determination. Measurements of the ferric ion level in the solutions have been performed in the presence of sulfosalicylic acid used as a substrate. The efficiency testing with the use of this method is based on determining the absorbance of ferric ions present in the solution after 24 hours of running the corrosion process. Its determination has been performed with the use of a spectrophotometer Marcel MINI. The amount of ferric ions in the solutions has been calculated using the standard curve. The corrosion inhibition efficiency has been calculated with the below given formula:

$$
E=\left(1-C_{\text {inh }} / C_{0}\right) * 100 \%
$$

where: $E$ - efficiency of the corrosion inhibition [\%], $C_{i n h}$ - concentration of ferric ions in the solution in the presence of a corrosion inhibitor $[\mathrm{mg} / \mathrm{L}], C_{0}-$ concentration of ferric ions in solution with no corrosion inhibitor applied $[\mathrm{mg} / \mathrm{L}]$.

\section{Results and discussion}

\subsection{Gravimetric method}

The obtained results are presented in the below given figures. The graphs show the corrosion process inhibition efficiency obtained in the presence of various THAM concentrations, its mixtures with thiourea and phosphorous acid, as well as with the thiourea solution.

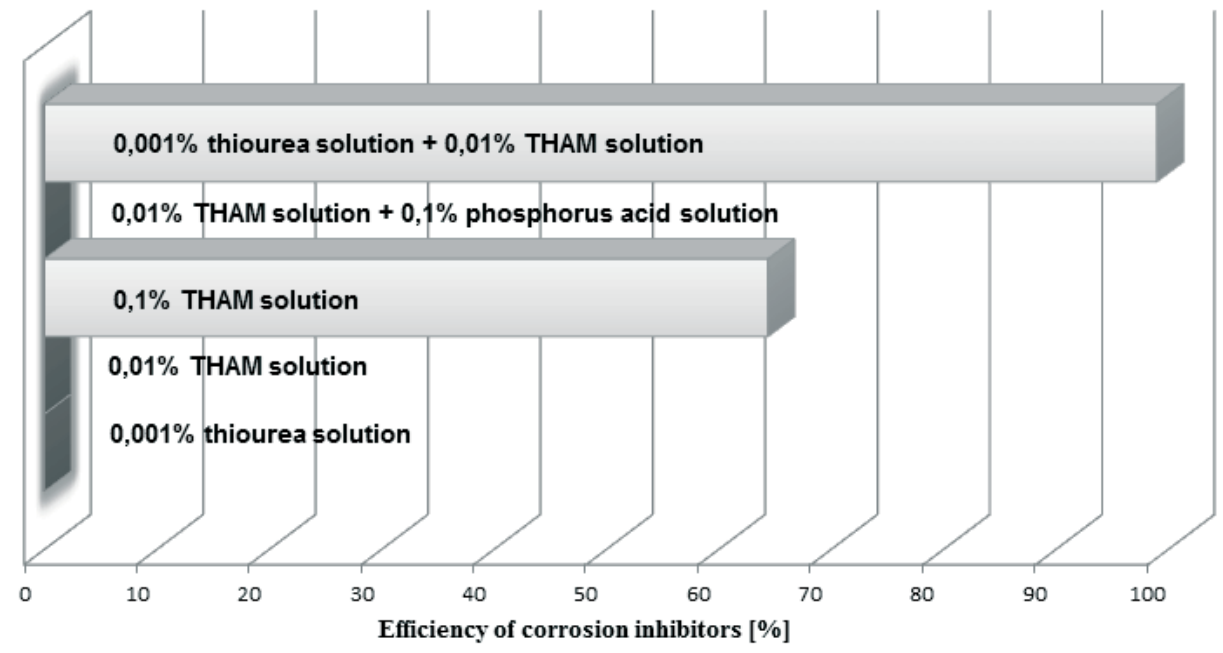

Fig. 1. Efficiency of the corrosion inhibition obtained for st3s steel 


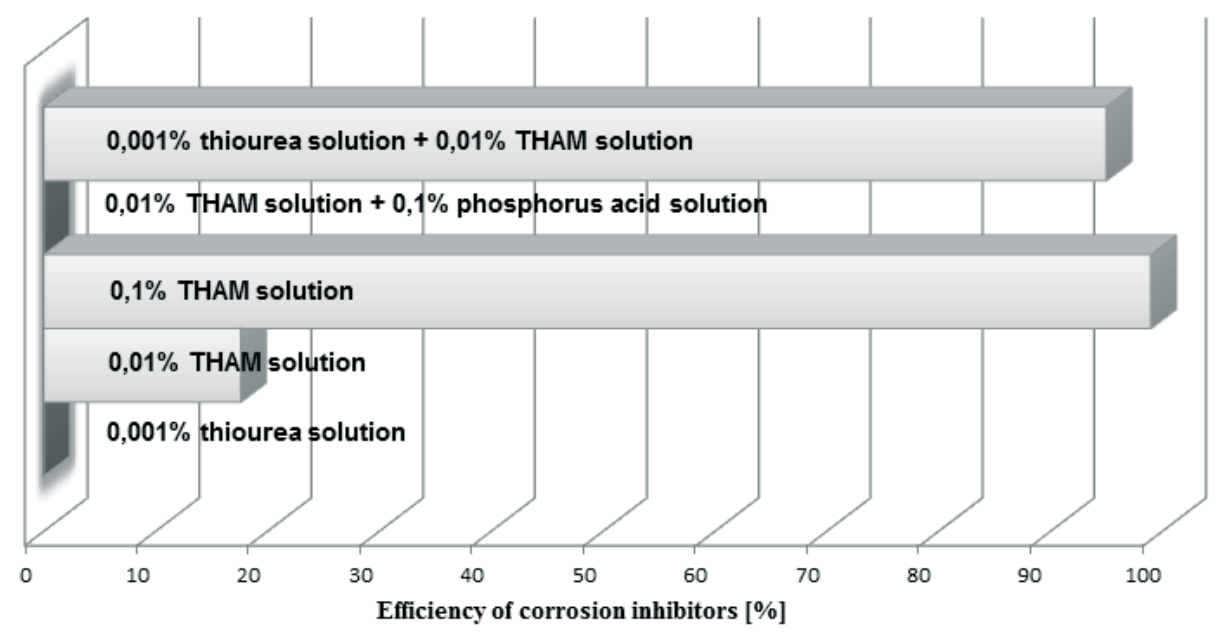

Fig. 2. Efficiency of the corrosion inhibition obtained for reinforcing steel

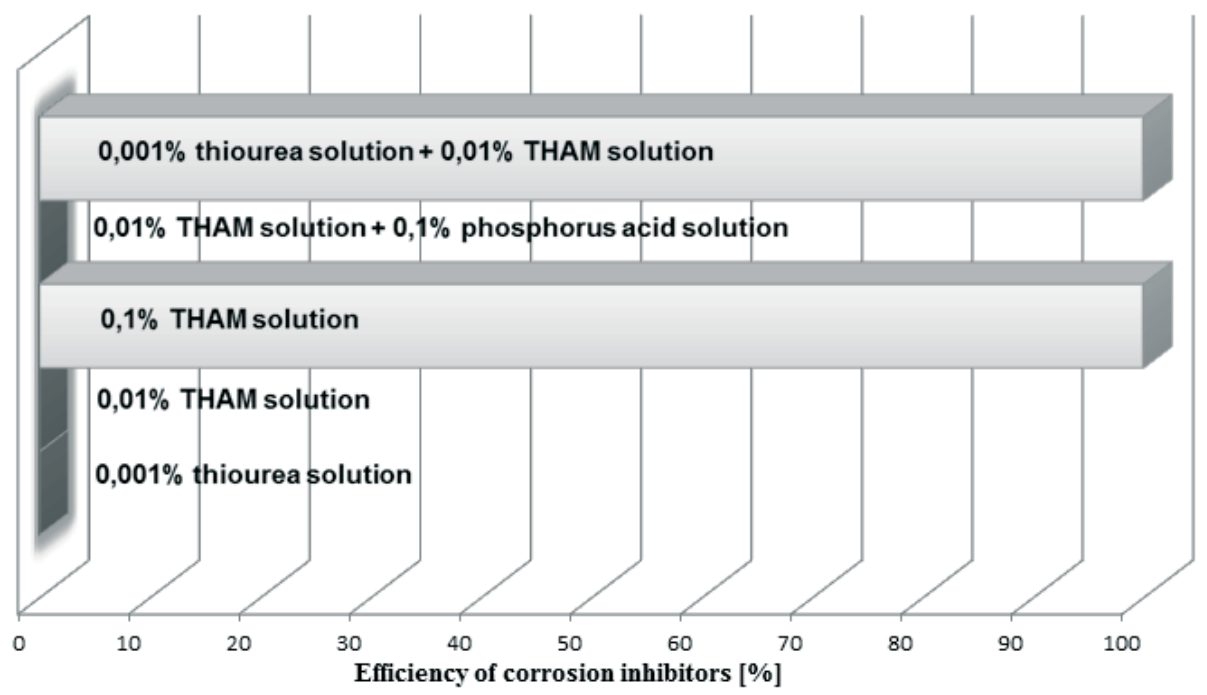

Fig. 3. Efficiency of the corrosion inhibition obtained for structural steel

Figure 2 presents an analysis of the corrosion process inhibition for the samples of carbon steel st3s. It shows that the THAM solution of the concentration $0.1 \%$ is a very efficient corrosion inhibitor in the presence of the corrosive calcium chloride solution. The highest efficiency of corrosion process inhibition on the surface of the st3s steel samples in the mentioned type of aggressive corrosive solution has been obtained with a mixture of the $0.001 \%$ thiourea solution and the $0.01 \%$ THAM solution. On the other hand the $0.001 \%$ thiourea solution, $0.01 \%$ THAM solution and its mixture with phosphorous acid have shown no corrosion inhibiting effect for those steel samples immersed in the aggressive solution of calcium chloride. An addition of the $0.001 \%$ thiourea solution to the $0.01 \%$ THAM solution brings about an increase of the corrosion inhibition by about $40 \%$.

Figure 3 is presents the corrosion inhibition effect obtained for reinforcing steel. As in the previous case, the THAM solution shows up to be a very good corrosion inhibitor. Its 
effect is stronger here than in the case of the st3s carbon steel samples. The corrosion process that occurs in the calcium chloride solution gets the most efficiently inhibited by the $0.1 \%$ THAM solution and its mixture with the $0.01 \%$ solution and the $0.001 \%$ thiourea solution. The corrosion inhibition effect decreases by about $85 \%$, when a ten-times weaker THAM solution is applied.

Figure 5 shows that in the case of structural steel the $0.1 \%$ THAM solution and its mixture with the $0.001 \%$ thiourea solution can also be successfully used as a corrosion inhibitor. The both mentioned solutions exhibit similar efficiency of the corrosion inhibition process. Weaker corrosion inhibitor solutions do not exhibit any protective properties in this case.

For all the above discussed cases concerning samples of st3s carbon steel, reinforcing steel and structural steel samples immersed in the calcium chloride solution, the $0.001 \%$ thiourea solution and the mixture of $0.01 \%$ THAM solution with the $0.1 \%$ phosphorous acid solution do not exhibit any corrosion inhibiting properties.

\subsection{Spectrophotometric method}

The efficiency obtained with the spectrophotometric method is higher as compared to the results obtained with the gravimetric method. A mixture of the $0.001 \%$ thiourea solution and the $0.01 \%$ THAM solution exhibits the best corrosion inhibiting properties for all the tested steel samples. This mixture inhibits the corrosion process at the efficiency approaching $100 \%$. Testing performed with the use of this method has yielded higher efficiency values for the $0.01 \%$ and $0.1 \%$ THAM solutions. In the case of the st $3 \mathrm{~s}$ and reinforcing steel samples, considerable corrosion inhibiting properties have been also observed for a mixture of the $0.01 \%$ THAM solution and the $0.1 \%$ phosphorous acid. On other hand, the $0.001 \%$ thiourea solution exhibits lower efficiency of the corrosion inhibition in all the analyzed cases. The fact that the gravimetric method application yields lower efficiency values can be explained by the formation of calcium chloride crystals on surfaces of the tested steel samples. It is the reason why reweighting the samples after the 24 hours of their being immersed in the corrosive solution can yield inaccurate results. Thus, the results obtained with the spectrophotometric method seem to be more reliable.

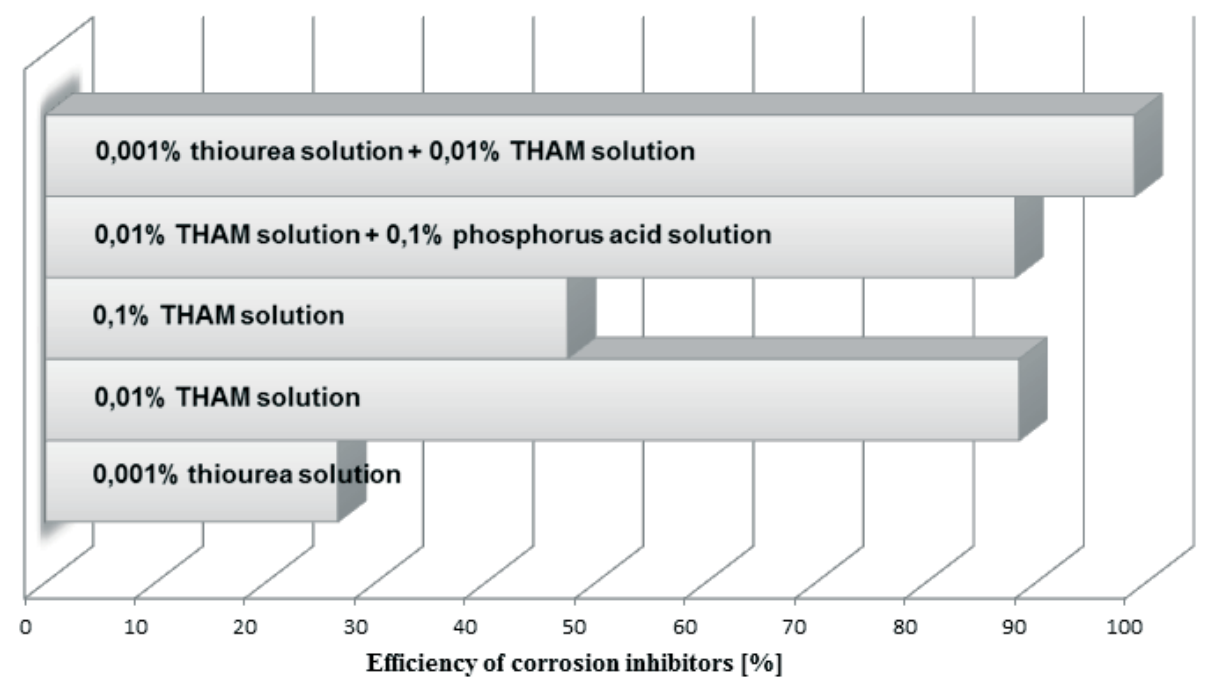

Fig. 4. Efficiency of the corrosion inhibition obtained for st3s steel 


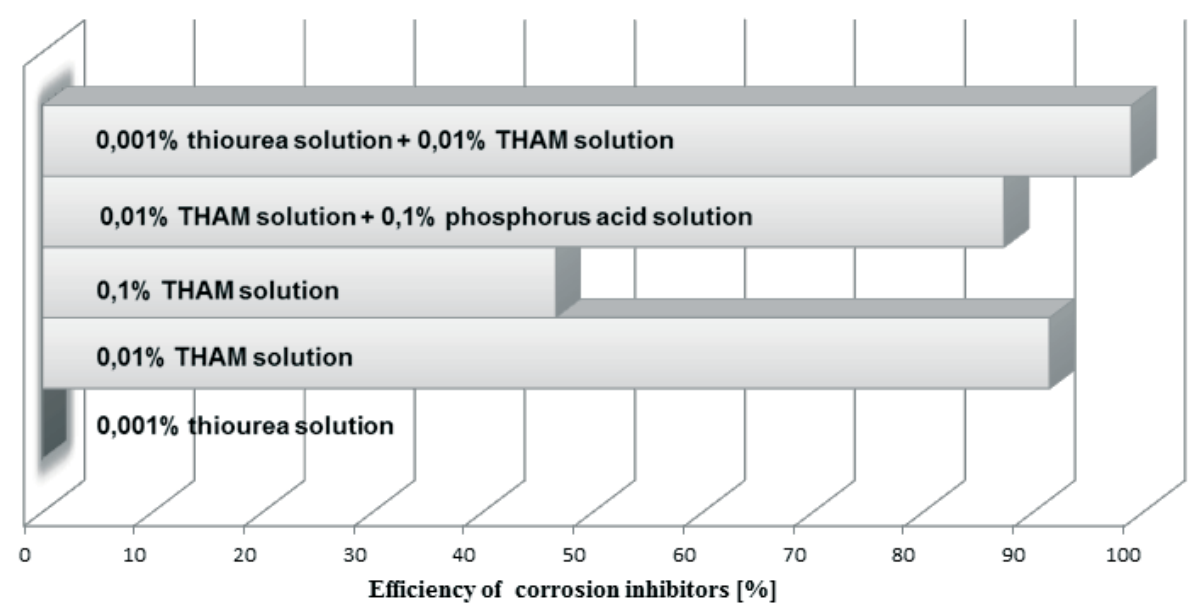

Fig. 5. Efficiency of the corrosion inhibition obtained for reinforcing steel

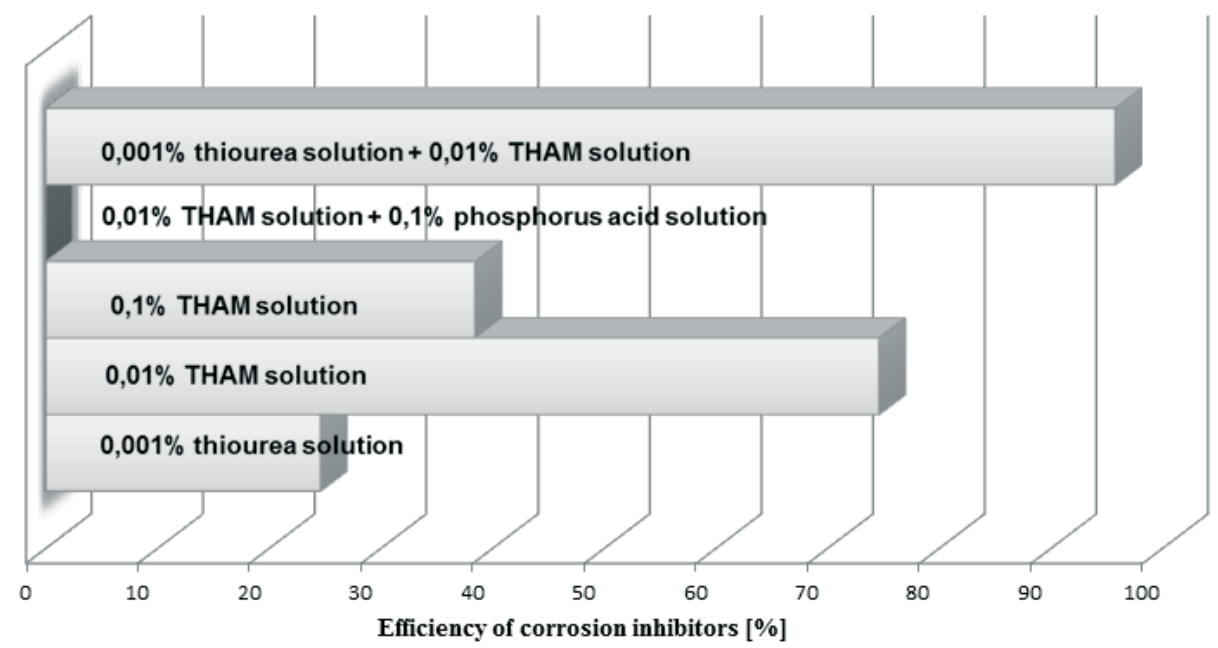

Fig. 6. Efficiency of the corrosion inhibition obtained for structural steel

\section{Conclusions}

- Thiourea solution that is commonly used to inhibit corrosion processes occurring in the calcium chloride solution can be successfully replaced by the THAM solution;

- It is important to find adequate corrosion inhibitors for the action of calcium chloride solution, as each winter enormous amounts of chlorides are used for ice removal from the roads

- Another important and actual objective of the discussed research area is to find new non-toxic and eco-friendly corrosion inhibitors;

- Although the THAM structure does not include any sulfur atom its corrosion inhibiting efficiency in $\mathrm{CaCl} 2$ is higher as compared to the thiourea solution;

- For carbon steel st3s samples, the $60 \%$, corrosion inhibition efficiency has been obtained with the $0.01 \%$ THAM solution and $20 \%$ - with the $0.1 \%$ THAM 
solution. When the $0.01 \%$ THAM solution has been added to the thiourea solution, the analyzed process has been inhibited in $70 \%$;

- For reinforcing steel samples immersed in the calcium chloride solution, no corrosion inhibition has been observed in the case of the $0.001 \%$ thiourea solution application;

- For of structural steel samples, the use of the 0.01\% THAM solution has yielded the corrosion inhibition efficiency of about $50 \%$ and in the case of the $0.1 \%$ THAM solution the obtained result has been of about $15 \%$. When the $0.01 \%$ THAM solution has been added to the $0.001 \%$ thiourea solution, the corrosion inhibiting efficiency has increased to about $70 \%$;

- In the case of the THAM solution application, the corrosion inhibition mechanism is probably related to the formation of its thin layer on metal surfaces.

- According to the literature data [3], an addition of phosphorous acid to a corrosive solution should increase the corrosion inhibiting effect. However, such a result has not been obtained in the testing performed for the discussed project.

We acknowledge support from Polish Ministry of Science and Higher Education within the statutory research number S/12/II B/2016.

\section{References}

1. Finsgar M., Milosev I. Inhibition of copper corrosion by 1,2,3-benzotriazole: A review. Corrosion Science 52 (2010) 2737-2749.

2. Tiu B.D.B., Advincula R. Polymeric corrosion inhibitors for oil and gas industry: Design principles and mechanism.

3. Finsgar M., Jackson J. Application of corrosion inhibitors for steels in acidic media for the oil and gas industry: A review. Corrosion Science 86 (2014) 17-41.

4. Verma Ch., Ebenso E.E., Bahadur I., Obot I.B., Quaraishi M.A. 5-(Phenylthio)-3H-pyrrole-4carbonitriles as effective corrosion inhibitors for mild steel in $1 \mathrm{M} \mathrm{HCl}$ : Experimantal and theoretical investigation. Journal of Molecular Liquids 212 (2015) 209-218.

5. Khadiri A., Saddik R., Bekkouche K., Aouniti A., Hammouti B., Benchat N., Bouachrine M., Solmaz R. Gravimetric, electrochemical and quantum chemical studies of some pyrizidine derivates as corrosion inhibitors for mild steel in $1 \mathrm{M} \mathrm{HCl}$ solution. Journal of the Taiwan Institute of Chemical Engineers 2015.

6. Mert B.D., Yuce A.O., Kardas G., Yazici B. Inhibition effect of 2-amino-4-methylpyridine on mild steel corrosion: Experimental and theoretical investigation. Corrosion Science 85 (2014) 287-295.

7. Zaferani S.H., Sharifi M., Zaarei D., Shishesaz M.R. Application of eco-friendly products as corrosion inhibitors for metals in acid picking processes - A review. Journal of Environmental Chemical Engineering 1 (2013) 652-657.

8. Soylev T.A., Richardson M.G. Corrosion inhibitors for steel in concrete: State-of-the-art report. Construction and Building Materials 22 (2008) 609-622.

9. Founda A.E.S., Etaiw S.E.H., El-Bendary M.M., Maher M.M. Metal-organic frameworks based on silver (I) and nitrogen donors as new corrosion inhibitors for copper in $\mathrm{HCl}$ solution. Journal of Molecular Liquids 213 (2016) 228-234.

10. Li X., Deng S., Fu H., Xie X. Synergestic inhibition effects of bamboo leaf extract/major components and iodide ion on corrosion of steel in H3PO4 solution. Corrosion Science 78 (2014) 29-42.

11. Negm N.A., Kandile N.G., Badr E.A., Mohammed M.A. Gravimetric and electrochemical evaluation of environmentally friendly nonionic corrosion inhibitors for carbon steel in $1 M$ $\mathrm{HCl}$. Corrosion Science 65 (2012) 94-103. 